\title{
Metodología para el Desarrollo de Sistemas de Combustión Sin Llama
}

\author{
Andrés A. Amell, Bernardo A. Herrera, Carolina Sepúlveda y Francisco J. Cadavid \\ Universidad de Antioquia, Facultad de Ingeniería, Grupo de Ciencia y Tecnología del Gas y Uso \\ Racional de la Energía- GASURE, Bloque 20, oficina 435, Calle 67, \# 53-108, Medellín-Colombia \\ (e-mail: anamell@udea.edu.co)
}

\begin{abstract}
Resumen
Este trabajo presenta el desarrollo de una metodología para el diseño de equipos de calentamiento de combustión sin llama. El diseño de equipos de combustión sin llama requiere de metodologías apropiadas que garanticen las condiciones necesarias para alcanzar este modo de combustión. No obstante, estas metodologías no se encuentran disponibles en la literatura científica. Para esto se ha empleado la combinación del escalamiento dinámico, en función de la relación de impulsos de aire/gas, y el uso de la simulación numérica con el software FLUENT® para obtener configuraciones y dimensiones geométricas adecuadas para la descarga del combustible y comburente. La metodología desarrollada ha sido aplicada en el diseño de un horno experimental de $20 \mathrm{~kW}$ que utiliza gas natural, verificándose experimentalmente su validez con la obtención del régimen de combustión sin llama de forma estable.
\end{abstract}

Palabras clave: combustión sin llama, equipos de calentamiento, transferencia de calor, simulación numérica

\section{Methodology to Develop Flameless Combustion Devices}

\section{Abstract}

A methodology to design flameless combustion heating equipment is presented. The design of flameless combustion devices requires an appropriate methodology to guarantee the necessary conditions to obtain this combustion mode. Nevertheless, such methodologies are not available in the scientific literature. For this, a combination of dynamic scaling, based on air/gas impulse ratio, and numerical simulation with FLUENT $®$ has been employed to get suitable geometric configurations and dimensions for discharging fuel and comburent air. The proposed methodology has been applied to the design of a $20 \mathrm{~kW}$ experimental furnace using natural gas as fuel. The validity of the methodology has been verified with stable flameless combustion operation in the furnace.

Key words: flameless combustion, heating equipment, heat transfer, numerical simulation 


\section{INTRODUCCIÓN}

La combustión sin llama es un novedoso y revolucionario tipo de combustión que fue descubierto en los años 90, obteniéndose cuando los impulsos de los flujos de gas combustible y aire inyectados a alta velocidad, generan corrientes de recirculación de los gases de combustión de moderada o alta intensidad, reduciéndose la concentración del oxígeno en la mezcla tripartita del combustible, el comburente y los gases recirculados y alcanzándose temperaturas mayores a la de autoignición del combustible (Yang y Blasiak, 2006). Se caracteriza porque la zona de reacción ocurre en todo el volumen de la cámara de combustión, sin la formación de frente de llama, ni ocurrencia de gradientes de temperatura significativos. Con la combustión sin llama se logra alcanzar simultáneamente alta eficiencia energética y muy reducidas emisiones de óxidos nitrosos (NOx) y monóxido de carbono (CO), así como calentamiento más uniforme, entre otras ventajas (Lupant et al., 2007; Szegö et al., 2008; Effuggi et al., 2008).

Si bien en los últimos diez años la combustión sin llama ha sido intensamente estudiada, aun su fenomenología y características no han sido del todo comprendidas (Effuggi et al., 2008; Szegö et al., 2008). Sin embargo, el desarrollo tecnológico de equipos de combustión y calentamiento que operan en este nuevo régimen de combustión se ha incrementado significativamente y cada día se identifican nuevos campos para su aplicación (Szegö et al., 2008). No obstante lo anterior, en la literatura no se encuentra una descripción sistemática y coherente de una metodología cuya aplicación garantice el desarrollo de equipos para la obtención de la combustión sin llama.

En este trabajo se desarrolla una metodología la cual permite obtener la combustión sin llama en equipos de combustión y calentamiento. La metodología desarrollada se fundamenta en considerar la relación de impulsos de las corriente de aire y combustible como parámetro de escalado dinámico y la simulación numérica con el software FLUENT® de las configuraciones geométricas que se van definiendo para verificar la obtención o no de la combustión sin llama.

\section{CONDICIONES PARA OBTENCIÓN DE LA COMBUSTIÓN SIN LLAMA}

Las condiciones fundamentales que deben ocurrir simultáneamente para obtener la combustión sin llama en una cámara de combustión son:

Una alta intensidad de recirculación de los gases de combustión que al mezclarse con los chorros de aire y gas, forman una mezcla tripartita donde la concentración de oxígeno debe estar entre $3 \%$ y $15 \%$, aproximadamente, antes de la reacción (Milani y Saponaro, 2001), con lo cual el tiempo característico de la reacción se incrementa y tiende a ser mayor al tiempo característico de mezclado, trayendo como consecuencia que la zona de reacción se extienda por el volumen de la cámara de combustión, obteniéndose una condición, en el diagrama de combustión de Borghi (Borghi y Champion, 2000), de reacción distribuida.

La temperatura de las paredes de la cámara de combustión debe permanecer mayor a la temperatura de autoignición del combustible que se está utilizando, así por ejemplo, para el metano cuya temperatura de autoignición es $580^{\circ} \mathrm{C}$, el rango mínimo de temperatura a que deben permanecer las paredes está entre $800^{\circ} \mathrm{C}$ y $850^{\circ} \mathrm{C}$ (Effuggi et al., 2008).

Para garantizar las condiciones óptimas de recirculación, se requiere que los impulsos del aire y el gas sean adecuados para obtener el factor de recirculación (Kv) necesario. Effuggi et al. (2008) encontraron que para generar el fenómeno de combustión sin llama se requieren factores de recirculación entre 4 y 10 . Este factor se define como el cociente entre el flujo másico de productos de combustión recirculados y la suma de los flujos másicos de aire y combustible.

Según la teoría de Curtet (1958), en una cámara de combustión, el factor de recirculación depende de los impulsos del aire, del gas combustible y de los gases de combustión que salen de la cámara, de acuerdo a la siguiente relación: 


$$
K_{v}=0.43\left(\sqrt{\frac{I_{g}}{I_{p}}+\frac{I_{a}}{2 I_{p}}-\frac{1}{2}}-1.65\right)
$$

\section{METODOLOGÍA PARA LA OBTENCIÓN DE LA COMBUSTIÓN SIN LLAMA}

En la Figura 1, se presenta un diagrama de flujo donde se ilustra el procedimiento seguido para desarrollar un sistema de combustión sin llama. El procedimiento se fundamenta en la combinación del criterio de escalado dinámico, al mantener constante la relación del impulso de los chorros de aire y gas, con la simulación numérica de los fenómenos térmicos y aerodinámicos en la cámara de combustión, para obtener los parámetros geométricos para el dimensionamiento del sistema de inyección de aire y gas, esto es, diámetro de las boquillas de descarga del aire, diámetro de la boquilla de descarga del gas, número de boquillas de aire, distribución perimetral de las boquillas de aire y posición relativa de éstas con respecto a la boquilla central de gas. Como criterios de verificación y validación del régimen de combustión sin llama, a parte de la inspección visual en los ensayos experimentales, se considera la uniformidad de los campos de temperatura, especies y flux de calor arrojados por la simulación numérica.

En este trabajo se propone como criterio de escalado dinámico mantener constante la relación entre el impulso de los chorros de aire y el impulso del chorro de gas combustible, de tal manera que a partir de una configuración conocida, donde se obtiene combustión sin llama, es posible encontrar las condiciones geométricas de descarga de los chorros de aire y gas para la nueva configuración, de acuerdo al siguiente algoritmo:

$$
\frac{I a}{I g}=\text { CONSTANTE }
$$

Obteniéndose a partir de la Ecuación (2), la siguiente expresión:

$$
\frac{\dot{m}_{a 1}{ }^{2}}{A_{a 1}} \cdot \frac{A_{g 1}}{\dot{m}_{g 1}{ }^{2}}=\frac{\dot{m}_{a 2}{ }^{2}}{A_{a 2}} \cdot \frac{A_{g 2}}{\dot{m}_{g 2}{ }^{2}}
$$

Si el combustible gaseoso, en ambas configuraciones, se inyecta a igual presión manométrica, se cumple la siguiente relación complementaria:

$$
\frac{P_{T 1}}{W_{1} A_{g 1}}=\frac{P_{T 2}}{W_{2} A_{g 2}}
$$

A partir de las ecuaciones (3) y (4) se obtienen entonces las áreas de las boquillas de inyección para el aire y el gas de la nueva configuración. Con estas dimensiones se procede a realizar el mallado de la nueva configuración para luego efectuar su simulación numérica con el objeto de verificar si se obtiene o no el fenómeno, antes de construir y probar experimentalmente.

\section{RESULTADOS Y DISCUSIÓN}

La metodología desarrollada se ha aplicado en el diseño del sistema de descarga de aire y gas, en una cámara de combustión de dimensiones $1350 \mathrm{~mm}$ x $600 \mathrm{~mm}$ x $600 \mathrm{~mm}$ de $20 \mathrm{~kW}$ de potencia, que utiliza como combustible gas natural, partiendo de la información de los flujos másicos y geometrías del quemador comercial autoregenerativo FLOX® REGEMAT (Lupant et al., 2007). En la Tabla 1 se presentan los parámetros característicos de ambas configuraciones. 


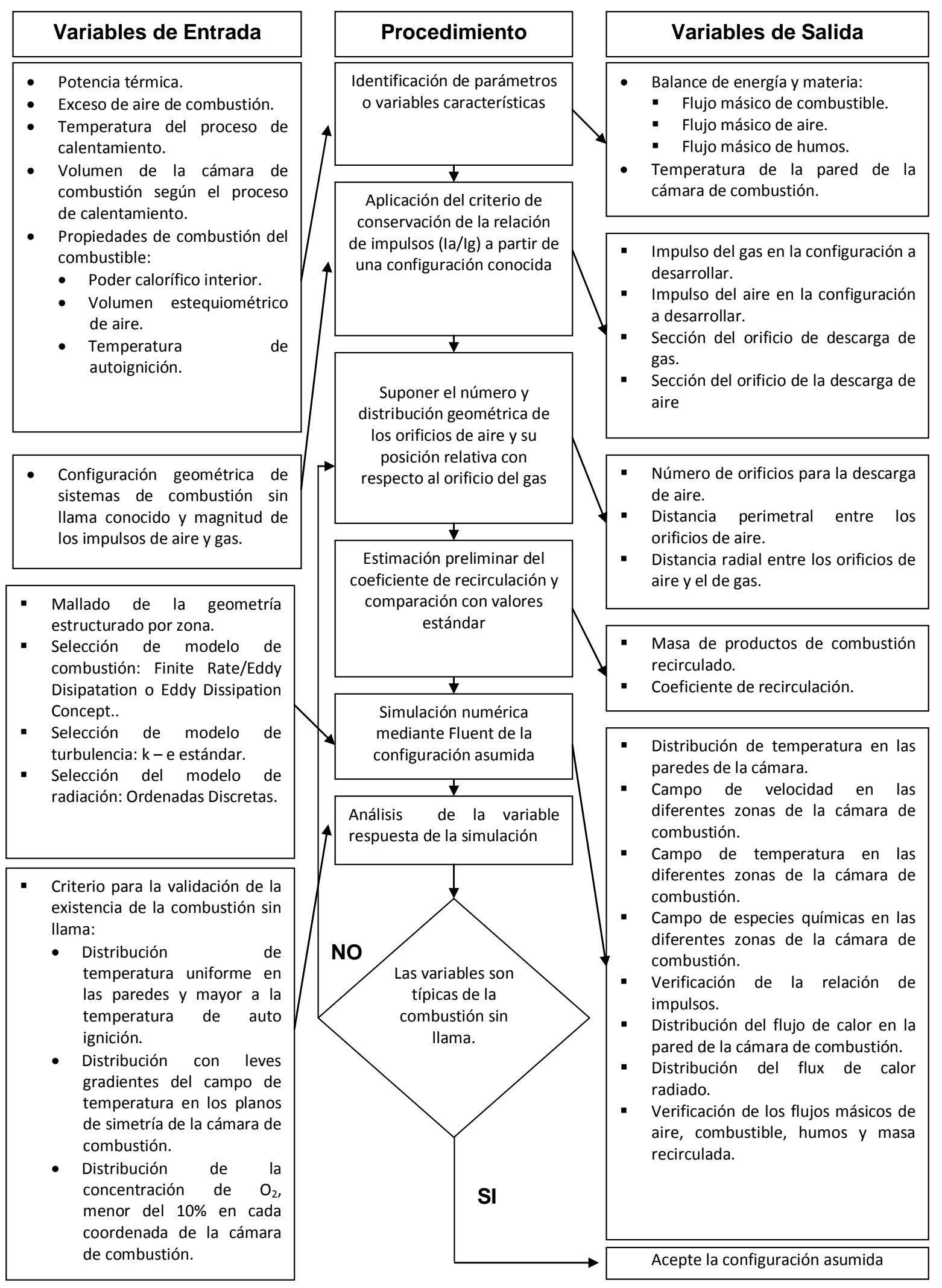

Fig. 1: Diagrama de flujo del procedimiento para desarrollar un sistema de combustión sin llama

Para la configuración de $20 \mathrm{~kW}$, se ha obtenido experimentalmente la combustión sin llama en condiciones de estabilidad. En la Figura 2, se muestra el perfil de temperatura obtenido a lo largo del eje de simetría de la cámara, mediante una sonda móvil de muestreo, como puede observarse, a partir de la coordenada de $300 \mathrm{~mm}$, punto en el que comienza aproximadamente la zona de reacción, la variación de la temperatura es leve, obteniéndose con ello una de las características de 
la combustión sin llama, también se observa una buena aproximación entre los datos de la simulación numérica y los datos experimentales.

Tabla 1: Parámetros característicos de las dos configuraciones.

\begin{tabular}{|l|c|c|c|}
\hline \multicolumn{1}{|c|}{ Datos de entrada } & & SISTEMA DESARROLLADO & REGEMAT \\
\hline Potencia térmica & $\mathrm{kW}$ & 20 & 200 \\
\hline Factor de aireación & - & 1.2 & 1.1 \\
\hline Combustible & - & Gas Natural & Gas Natural \\
\hline $\begin{array}{l}\text { Radio desde el centro del quemador hasta el } \\
\text { centro de los puertos de aire }\end{array}$ & $\mathrm{mm}$ & 120 & 170 \\
\hline Temperatura de entrada del aire de combustión & ${ }^{\circ} \mathrm{C}$ & 600 & 800 \\
\hline Diámetro de los puertos de combustible & $\mathrm{mm}$ & 3.19 & 13 \\
\hline Diámetro de los puertos de aire & $\mathrm{mm}$ & 16 & 20 \\
\hline Velocidad del combustible & $\mathrm{m} / \mathrm{s}$ & 90 & 50 \\
\hline Velocidad del aire & $\mathrm{m} / \mathrm{s}$ & 70 & 120 \\
\hline Impulso del aire & $\mathrm{N}$ & 0.29 & 1.58 \\
\hline Impulso del gas & $\mathrm{N}$ & 0.036 & 0.21 \\
\hline Relación de impulsos & - & 8 & 8 \\
\hline
\end{tabular}

En la Figura 3, se presenta la distribución de temperatura en la pared superior, mostrándose un alto grado de uniformidad y valores superiores a la temperatura de autoignición del gas natural en la región posterior a los $300 \mathrm{~mm}$ de longitud de la cámara de combustión, donde probablemente se desarrolla la zona de reacción. Esta uniformidad se verifica con el Factor de Uniformidad Térmica $\mathrm{R}_{\mathrm{tu}}$, el cual tiene un valor de 0.18 , cercano a cero que es el valor ideal para una uniformidad total. La expresión para el cálculo de $\mathrm{R}_{\mathrm{tu}}$ está dada por la ecuación (5).

$R_{t u}=\sqrt{\sum\left(\frac{T_{i}-\bar{T}}{\bar{T}}\right)^{2}}$

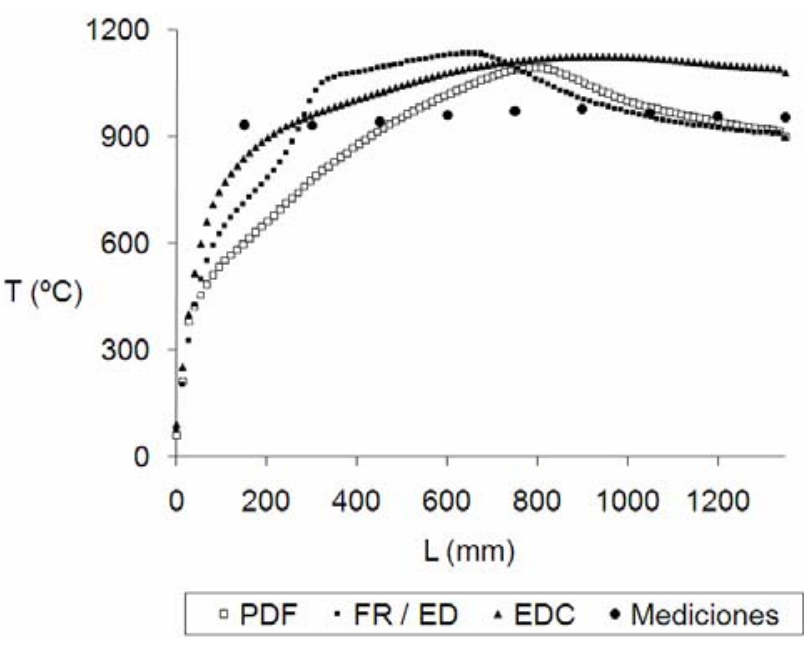

Fig. 2: Comportamiento de la temperatura a lo largo del eje de simetría de la cámara en régimen de combustión sin llama.

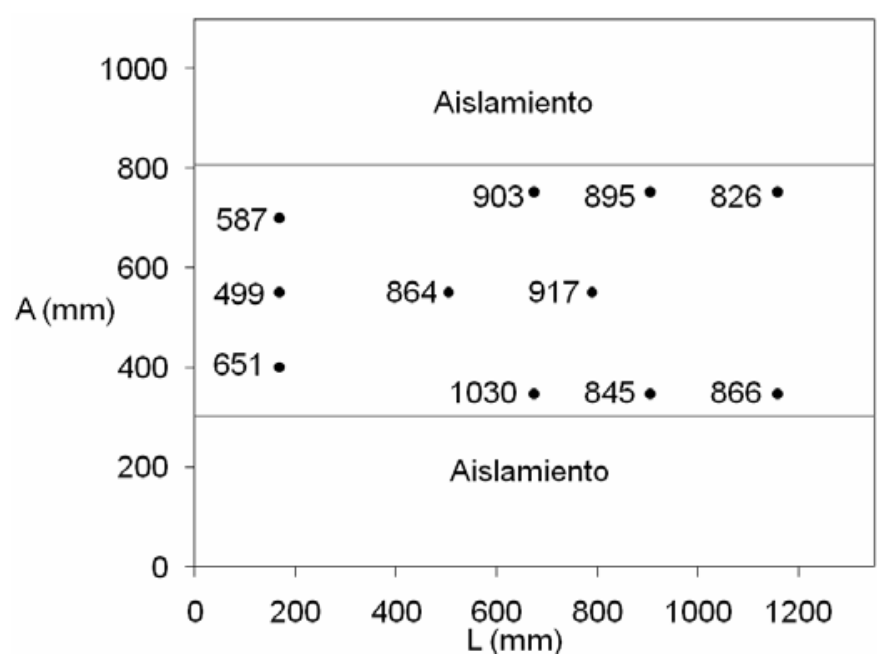

Fig. 3: Comportamiento de la temperatura de pared de la cámara en régimen de combustión sin llama.

\section{CONCLUSIONES}

Se ha desarrollado una metodología para el diseño de sistemas de inyección de combustible y aire para obtener un régimen de combustión sin llama, en una cámara de combustión de volumen y 
capacidad de calentamiento conocida, a partir de la combinación del criterio de relación constante de impulsos de aire y gas, y la simulación numérica utilizando el software FLUENT.

La validez predictiva de la metodología desarrollada para obtener parámetros y configuraciones geométricas óptimas para la descarga de los chorros de aire y gas, ha sido probada en el diseño y evaluación experimental, de una cámara de combustión, operando en régimen de combustión sin llama de $20 \mathrm{~kW}$ de potencia térmica.

\section{NOMENCLATURA}

A Ancho de la cámara de combustión (mm)

$\mathrm{A}_{\mathrm{g}} \quad$ Área de la boquilla de descarga de gas $\left[\mathrm{m}^{2}\right]$

$A_{a} \quad$ Área de la boquilla de descarga de aire $\left[\mathrm{m}^{2}\right]$

$\mathrm{P}_{\mathrm{t}} \quad$ Potencia térmica de la cámara de combustión [kW].

W Índice de Wobbe del combustible usado $\left[\mathrm{kWh} / \mathrm{m}^{3}\right]$.

$\dot{m} \quad$ Flujo másico $[\mathrm{kg} / \mathrm{s}]$

I Impulso [N]

$K_{v} \quad$ Factor de recirculación

$m \quad$ Parámetro de Craya y Curtet

$T_{i} \quad$ Temperatura de la pared en el punto $i$
$\bar{T} \quad$ Temperatura media de pared

Subíndices

a Aire

$g$ Gas

p Productos de combustión

1 Parámetro o condición en la configuración de referencia.

2 Parámetro o condición en la configuración a desarrollar.

\section{AGRADECIMIENTOS}

Los autores agradecen a Colciencias por la financiación de la investigación sobre combustión sin llama, de cuyos resultados hace parte este trabajo.

\section{REFERENCIAS}

Borghi R. y M. Champion; Modélisation et théorie des flammes. 245-246. Éditions Technip Paris, Francia (2000).

Curtet, R.; Confined jets and recirculation phenomena with cold air. Combustion and Flame: 2, 383411 (1958).

Effuggi, A., D. Gelosa, M. Derudi y R. Rota; Mild combustion of methane-derived fuel combustion of methane-derived fuel mixtures: natural gas and biogas. Combustion Science and Technology: 180, 481-493 (2008).

Lupant, D., B. Pesenti, P. Evrard y P. Lybaert; Numerical and experimental characterization of a selfregenerative flameless oxidation burner operation in a pilot-scale furnace. Combustion Science and Technology: 179, 437-453 (2007).

Milani, A. y A. Saponaro; Diluted Combustion Technologies. IFRF Combustion Journal: No.200101 (2001).

Szegö, G. G., B. B. Dally y G.J. Nathan; Operational characteristics of a parallel jet MILD combustion burner system. Combustion and Flame: 152(2), 429-438 (2008)

Yang, W. y W. Blasiak; CFD as applied to high temperatura air combustion in industrial furnaces. IFRF Combustion Journal: No.200603 (2006). 